Continuum Mech. Thermodyn. 7 (1995) 1-22

$\overline{\text { Continum Mechanics }}$ Thermodynamics

(C) Springer-Verlag 1995

\title{
On constitutive equations for electrorheological materials
}

\author{
Alan S. Wineman and K. R. Rajagopal
}

Constitutive equations for electrorheological (ER) fluids have been based on experimental results for steady shearing flows and constant electric fields. The fluids have been modeled as being rigid until a yield stress is reached. Additional stress is then proportional to the shear rate. Recent experimental results indicate that ER materials have a regime of solid-like response when deformed from a rest state. They behave in a viscoelastic-like manner under sinusoidal shearing and exhibit time-dependent response under sudden changes in shear rate or electric field. In this work, a constitutive theory for ER materials is presented which accounts for these recent experimental observations. The stress is given by a functional of the deformation gradient history and the electric field vector. Using the methods of continuum mechanics, a general three-dimensional constitutive equation is obtained. A sample constitutive equation is introduced which is then used to determine the response of an ER material for different shear histories. The calculated shear response is shown to be qualitatively similar to that observed experimentally.

\section{Introduction}

Much of the research and development work on electrorheological (ER) materials has been concerned with one-dimensional shearing deformations in the presence of an electric field. Even though an ER material is a suspension of electrically non-conducting particles in a non-conducting liquid, the response of an ER material in such deformations is usually described in terms of an equivalent single - constituent material. Thus, in steady one-dimensional shearing flows, which has been the subject of most studies, an ER material is generally regarded as a Bingham fluid whose yield stress and viscosity depend on the strength of the electric field. In this manner, yield stress and viscosity have become characteristic material properties of ER materials and much emphasis has been placed on their measurement. 
The constitutive equations which have been proposed for ER fluids have been based on results for steady shearing flows, in which the constitutive equation takes the form of the scalar shear stress-shear rate relation for a Bingham fluid. For three dimensional flows, Atkin, Shi and Bullough [1] assumed the constitutive equation to be that for a Bingham fluid. This model is essentially obtained from the one - dimensional case by generalizing the scalar shear stress and shear rate to the stress and stretching tensors, respectively, and the yield stress to a stress dependent yield condition. Rajagopal and Wineman [2] adopted a completely different approach in the development of a constitutive equation for three dimensional flows. They assumed that the stress tensor depends on the electric field vector and stretching tensor, all at the same instant. Utilizing the methods of continuum mechanics, they developed a general form for this dependence. In the case of a simple shear flow, the constitutive equation gives the shear stress in terms of the shear rate and electric field. One particular choice of this relation can be that for a Bingham fluid. It also predicts the presence of normal stresses due to the electric field vector and the interaction between the electric field vector and the shear field.

Recent research has been concerned with the response of ER materials when sinusoidal shearing deformations have been imposed (see for example, Brooks et al [3], Vinogradov et al [4], Thurston and Gaertner [5], Xu and Liang [6], Yen and Achorn [7], Gamota and Filisko [8], [9], Jordan and Shaw [10]). According to the experimental results, when the strain amplitudes are sufficiently small, the shear stress is also sinusoidal and is out of phase with the strain. The stress is expressed in a manner analogous to that in linear viscoelasticity, that is, in terms of an elastic storage modulus (real part) and a loss modulus (imaginary part) of a complex modulus. These moduli appear to depend weakly on frequency and strongly on the electric field. Gamota and Filisko [8] have studied the effect of increasing the strain amplitude. They showed that the response becomes nonlinear in the sense that the stress response becomes non-sinusoidal, but remains periodic. Yen and Achorn [7] and Jordan and Shaw [10] report similar observations. Gamota and Filisko [8] also showed that nonlinearities arise in the stress response as the electric field increases. Gamota et al [11], in a Fourier decomposition of the stress response, showed that the nonlinearities can be attributed to the superposition on the small strain response of sinusoidal oscillations whose frequencies are odd multiples of the fundamental frequency. The third 'overtone' appears when the electric field becomes sufficiently strong. Increasing the electric field introduces the fifth 'overtone', etc.

In linear viscoelasticity, the complex modulus which characterizes the response to sinusoidal strains can be related to time dependent phenomena associated with creep and stress relaxation. It is natural to inquire whether the same is true for ER materials. The first study of this possibility appears to be that of $\mathrm{Xu}$ and Liang [6]. In a study of start-up flows under constant shear strain rate, they showed that the shear stress grows rapidly and continuously to the steady state value that would occur under steady shear flow. The details of the stress growth history depend on the electric field. The stress growth is similar in form to that which would be found for viscoelastic fluids. Other authors have also presented evidence of transients in the stress response of ER materials, although 
this has appeared in the context of studies of other phenomena. Thurston and Gaertner [5] showed the response to a step change on and off for a dc electric field. Conrad et al [12] and Jordan et al [10] showed results for start up flows under constant shear rate. Their results are similar to those of Xu and Liang [6].

The results of the sinusoidal strain and constant strain rate experiments are not accounted for by the constitutive equations discussed above. The purpose of the present work is to discuss possible forms for constitutive equations which overcome this deficiency. In section 2 we introduce constitutive assumptions which are motivated by these experiments. A constitutive theory is presented in which the current stress depends on the history of deformation and the current electric field vector. A specific example of a constitutive equation is introduced in Section 3, while the general representation of the constitutive equation in terms of kinematic tensorial quantities and the electric field vector is presented in the Appendix. General simple shear histories are introduced in Section 4. Expressions are then developed for the shear and normal stress components based on the constitutive equation of Section 3. Section 5 contains a study of the response under start-up flow at a constant shear rate. Sinusoidal shear is discussed in Section 6. It is shown that the results based on the sample constitutive equation can represent the gross features of the experimental observations discussed above. This implies that the constitutive equation for a specific ER material can be developed using the constitutive frame work presented here.

\section{Constitutive assumptions}

We begin this section with a discussion of a number of aspects of the response of ER materials.

(a) Consider an ER material which is contained between two parallel plates. Let the material initially be at rest in an unstressed state, and let there be no electric field. If a shear stress is applied, the material will undergo shear flow, acting as a fluid. Next let the ER material be placed in an electric field and then let a shear stress be applied. If the shear stress is small enough, the ER material develops sufficient stiffness and strength so that the ER material can resist flow and respond as a solid. The ER material appears to have solidified by the application of the electric field. That is, the ER material is in a state in which it can support a constant shear stress when it has undergone a constant shear strain from the state at which it solidified. The shear stress will depend on the shear strain and the strength of the electric field.

Under a sufficiently large shear stress, the ER material loses its ability to act as a solid. The material will undergo shear flow and thereby respond as a fluid. The shear stress will become independent of the shear strain and become dependent instead on a kinematical quantity which is appropriate to the description of its response as a fluid, as well as the strength of the electric field. In other terms, distortions from the configuration at solidification have decreasing influence on the stress, and hence the material 'loses memory' of that configuration. The manner in which the ER material undergoes the transition from a solid-like to 
fluid-like response is currently the subject of research activity and will not be speculated on here. The approach here only makes use of the observation that when the shear stress reaches some value, there appears to be such a transition.

(b) The preceding discussion describes the response as the shear stress is increased from a value below that at transition to a value above. Now consider the reverse situation in which the shear stress is reduced from a value above that at transition to a smaller one. There do not appear to be any studies of this process in the literature which can be used to guide a constitutive assumption for the subsequent response. In order to provide the starting point for such a study, the response of the material is assumed to be as follows. When the shear stress has decreased to the transition value, the ER material begins to act as a solid. This means that the stress becomes dependent on the strain from some configuration. The configuration of the material at the time when the shear stress equals the transition stress becomes the reference configuration from which strains are measured. Although the strains are zero at the moment of transition, the stresses are not. It is assumed that the stress is continuous at transition.

(c) Consider steady shear flow in which the electric field vector is normal to the fluid velocity. For such conditions, the constitutive equations discussed in the Introduction give relations of the form

$\sigma(t)=f(\gamma(t)), E(t))$,

where $\sigma(t), E(t)$ and $\gamma(t)$ denote, respectively, the values at time $t$ of the shear stress, electric field strength and shear rate. Now consider deformation control experiments in which the shearing deformation is sinusoidal, i. e. $\gamma(t)=$ $\gamma_{0} \sin \omega t$. If the material is modeled as a Bingham material, equation (2.1) applies only if a yield stress is exceeded. It is then unclear how deformation control experiments are to be described. In the approach of Rajagopal and Wineman [2], (2.1) is a general form which can apply under all flow conditions. According to this constitutive equation, the shear stress is in phase with the shear rate under sinusoidal deformations. The shear stress will be sinusoidal if the amplitude of the shear rate, $\gamma_{0}$, is sufficiently small. As $\gamma_{0}$ increases, the stress response becomes non-sinusoidal.

In order that it be able to describe a phase difference between the stress and strain, (2.1) must be modified. Constitutive equations for linear viscoelasticity, which are known to account for phase differences between stress and strains during sinusoidal oscillations, suggest appropriate mathematical modifications. The constitutive equation for ER materials will depend on some aspect of the deformation history, such as on a time derivative of the strain or strain rate or on an integral over the strain history.

(d) The response of ER materials to changes in the electric field is almost instantaneous. Thus, while the stress depends on the history of the deformation, it also depends on the instantaneous value of the electric field.

The preceding discussion is now incorporated into a constitutive theory for ER materials. The ER material is modeled as a single constituent material. As a prelude to stating the constitutive equation for three dimensional response, it is 
useful to consider one-dimensional homogeneous shear deformations in which the electric field vector is normal to the direction in which the material displaces, or flows. The notation introduced for (2.1) is retained here. Let $\sigma_{0}(E)$ denote the shear stress corresponding to electric field $E$ when the material undergoes the transition from solid-like to fluid-like response, as discussed in (a). Possible time variations are shown in figure 1 , of the electric field $E(t)$, the shear stress $\sigma_{0}(E(t))$ required for transition, and the actual shear stress in the material $\sigma(t)$. The constitutive equation is defined in a piece-wise manner, in which its form depends on whether the response is solid-like or fluid-like.

Now consider the three dimensional formulation and let $\sigma(t)$ and $\mathbf{E}(t)$ denote the stress tensor and electric field vector, respectively, at time $t$. It is assumed that the regimes of solid and fluid response of the ER material can be characterized by means of a transition function, $A(\sigma(t), \mathbf{E}(t))$ which has the following properties:

transition between solid and fluid solid regime

fluid regime

$$
\begin{aligned}
& A(\sigma(t), \mathbf{E}(t))=0, \\
& A(\sigma(t), \mathbf{E}(t))<0, \\
& A(\sigma(t), \mathbf{E}(t))>0 .
\end{aligned}
$$

If the only non-zero stress component is $\sigma_{12}=\sigma$, and the only component of the electric field vector is $E_{2}=E$, as in Figure 1, (2.2a) reduces to the condition $\sigma(t)=\sigma_{0}(E(t))$, provided $A$ has the right properties. It is also possible that $A$ is itself a functional. However, here we shall assume that $A$ is a function. It would be appropriate at this juncture to tie up the above modeling ideas with approaches that have been employed previously. The modeling outlined in this paper can be cast within the framework of the ideas espoused by Rajagopal and Wineman [13] in their work on constitutive theories and branching of response based on the notion of selectivity, the selection criterion being the transition function $A$. The recent papers by Wineman and Rajagopal [14] and Rajagopal and Wineman [15] on inelastic response of materials also bear a close relation, as far as the seminal ideas are concerned, and here we have to associate the activation criterion used in those papers with the transition function $A$.

Let $\left[t_{0}, t_{1}\right]$ denote the initial time interval when the response is solid-like, so that condition (2.2b) is met. It is assumed that the stress depends on the electric field at time $t$ and the history of the deformation from the configuration when the material solidifies. This is taken as the configuration at time $t_{0}=0$, which acts as a reference configuration for the newly formed solid material. The stress is given by

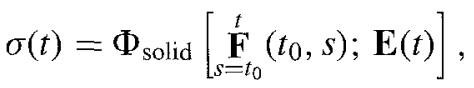

in which $\mathbf{F}\left(t_{0}, s\right)$ denotes the deformation gradient of the configuration at time $s$ with respect to the configuration at time $t_{0}$. It is assumed that the ER material acts as an isotropic solid. This constitutive relation must meet the restrictions imposed by material frame indifference and material isotropy. It can be shown by standard arguments in continuum mechanics, that (2.3) can be written in the 
form

$\sigma(t)=\Phi_{\text {solid }}\left[\mathbf{B}\left(t_{0}, t\right), \underset{s=t_{0}}{\mathbf{C}}(t, s) ; \mathbf{E}(t)\right]$,

where $\mathbf{B}\left(t_{o}, t\right)=\mathbf{F}\left(t_{0}, t\right) \mathbf{F}\left(t_{0}, t\right)^{T}$ and $\mathbf{C}(t, s)=\mathbf{F}(t, s)^{T} \mathbf{F}(t, s)$. Although the functionals in (2.3) and (2.4) are different, they are denoted by the same symbol for the purpose of simplicity of notation. It can also be shown that $\Phi_{\text {solid }}$ is an isotropic function of its arguments.

Let $\left[t_{1}, t_{2}\right]$ denote the next time interval, in which the material acts as a fluid, so that $(2.2 \mathrm{c})$ is satisfied. Since the particulate structure of the ER material has lost its ability to act as a solid, it is assumed that deformations from the configuration at solidification no longer affect the stress. As in the theory of simple fluids, the stress is now determined by the history of the deformation relative to the current configuration and the electric field,

$\sigma(t)=\Phi_{\text {fluid }}\left[\underset{s=t_{1}}{\stackrel{t}{\mathbf{F}}}(t, s) ; t_{1} ; \mathbf{E}(t)\right]$

It is a consequence of material frame indifference that (2.5) becomes

$\sigma(t)=\Phi_{\text {fluid }}\left[\underset{s=t_{1}}{\mathbf{C}}(t, s) ; t_{1} ; \mathbf{E}(t)\right]$

and is an isotropic functional of its arguments. Again, the same notation for the functional has been used in (2.5) and (2.6) for the sake of simplicity.

Note the explicit dependence on time $t_{1}$ when there is transition from solidlike to fluid-like response. This is included so as to ensure continuity of stress at the transition. Thus, if the response functionals in (2.4) and (2.6) are evaluated at $t=t_{1}$ and then equated, the following statement of continuity of the stress at $t_{1}$ is obtained:

$\Phi_{\text {solid }}\left[\mathbf{B}\left(t_{0}, t_{1}\right), \underset{s=t_{0}}{\mathbf{C}}\left(t_{1}, s\right) ; \mathbf{E}\left(t_{1}\right)\right]=\Phi_{\text {fluid }}\left[\underset{s=t_{1}}{t_{1}}\left(t_{1}, s\right) ; t_{1} ; \mathbf{E}\left(t_{1}\right)\right]$

Let $\left[t_{s i}, t_{s j}\right]$ be any time interval in which $(2.2 \mathrm{~b})$ is satisfied. It is assumed that the material solidifies at time $t_{s i}$, and its configuration at this time acts as a reference configuration. The stress is given by

$\sigma=\Phi_{\text {solid }}\left[\mathbf{B}\left(t_{s i}, t\right), \underset{s=t_{s i}}{\mathbf{C}}(t, s) ; t_{s i} ; \mathbf{E}(t)\right]$ 
Let $\left[t_{f i}, t_{f j}\right]$ be any time interval in which $(2.2 \mathrm{c})$ is satisfied. It is assumed that the material again becomes fluid-like with the stress given by

$\sigma=\Phi_{\text {fluid }}\left[\underset{s=t_{f i}}{\mathbf{C}}(t, s) ; t_{f_{i}} ; \mathbf{E}(t)\right]$

The dependence on $t_{s i}$ in (2.8) and on $t_{f i}$ in (2.9) is included to ensure continuity of the stress at transition, so that relations such as (2.7) are satisfied.

The transition function $A(\sigma, \mathbf{E})$ in $(2.2)$ is also subject to the restrictions of material frame indifference and material symmetry. The focus in this paper is on the response functionals $\Phi_{\text {solid }}$ in (2.4) and $\Phi_{\text {fluid }}$ in (2.6). Consequently, the restrictions on $A(\sigma, \mathbf{E})$ will not be pursued here.

The general forms of the response functionals $\Phi_{\text {solid }}$ in (2.4) and $\Phi_{\text {fluid }}$ in (2.6), as restricted by isotropy, can be determined by the methods presented in the article by Spencer [16]. This is discussed further in the Appendix.

We conclude this section with several comments. In a general three-dimensional formulation, we could define the stress through

$\sigma=\stackrel{\Phi}{\Phi=0}_{s=0}^{\infty}[\mathbf{F}(t-s) ; \mathbf{E}(t-s)]$

where $\mathbf{F}(t-s)$ is the history of the deformation gradient and $\mathbf{E}(t-s)$ is the history of the electric field. In the absence of the electric field, the constitutive relation reduces to that for a simple material. Depending on the histories of the deformation gradient and the electric field, it is possible that the functional $\Phi$ could represent fluid or solid response at a material point in the body. Here, for the purpose of simplicity of exposition, the solid and fluid like behavior in the different regimes of response is made explicit from the outset. When the response is that of a solid, then $\Phi$ depends on the history of the strain tensor referred to the reference configuration, and when the response is fluid-like $\Phi$ depends on the history of the strain tensor referred to the current configuration.

The above formulation defines an explicit transition of the ER material from apparent solid-like to fluid-like response. It is possible that as the transition function $A$ changes from negative to positive, $\Phi_{\text {solid }}$ in $(2.4)$ becomes independent of $\mathbf{B}(0, t)$ in a continuous, but rapid manner. The rapid but continuous transition of the ER material from solid-like to fluid response then corresponds to a similar transition from $\Phi_{\text {solid }}$ in (2.4) to $\Phi_{\text {fluid }}$ in (2.6).

\section{Constitutive example}

The representations of $\Phi_{\text {solid }}$ in (2.4) and $\Phi_{\text {fluid }}$ in (2.6) presented in the Appendix, are expressed in terms of general functionals of matrix polynomials of the kinematic tensors $\mathbf{B}(0, t)$ and $\mathbf{C}(t, s)$ and the electric field vector $\mathbf{E}(t)$. It is useful to present an example of a constitutive equation which illustrates possible forms for the functionals. This example constitutive equation will also be 
used, in section 4 , in the calculation of stresses in shear flows. Its purpose is to show the contribution to the stress components of the interaction between the kinematic tensors $\mathbf{B}(0, t)$ and $\mathbf{C}(t, s)$ and the electric field vector $\mathbf{E}(t)$.

It is to be emphasized that the following constitutive example is presented only for the purpose of illustrating the general model. It is not proposed as one for any particular ER material, as that would have to be determined on the basis of an extensive experimental program. The results in section 4 based on this example, may provide some useful ideas to be employed in the development of an appropriate constitutive equation for a specific material.

Attention is restricted to incompressible ER materials. For times $t \in\left[t_{0}, t_{1}\right]$, when the response is solid-like, the response functional in (2.4) is assumed to have the form

$\Phi_{\text {solid }}+p \mathbf{I}=\hat{\Phi}$

$=\beta_{1} \mathbf{E} \otimes \mathbf{E}+\beta_{2} \hat{\mathbf{B}}+\beta_{3} \hat{\mathbf{B}}^{2}+\beta_{4} \mathbf{D}$

$+\beta_{5}(\hat{\mathbf{B}} \mathbf{E} \otimes \mathbf{E}+\mathbf{E} \otimes \hat{\mathbf{B}} \mathbf{E})+\beta_{6}\left(\hat{\mathbf{B}}^{2} \mathbf{E} \otimes \mathbf{E}+\mathbf{E} \otimes \hat{\mathbf{B}}^{2} \mathbf{E}\right)$

$+\beta_{7}(\mathbf{D E} \otimes \mathbf{E}+\mathbf{E} \otimes \mathbf{D E})$

$+\int_{0}^{t} L_{1}(t-s) \hat{\mathbf{C}}(t, s) d s$

$+\int_{0}^{t} \int_{0}^{t} L_{2}\left(t-s_{1}, t-s_{2}\right)\left[\hat{\mathbf{C}}\left(t, s_{1}\right) \hat{\mathbf{C}}\left(t, s_{2}\right)+\hat{\mathbf{C}}\left(t, s_{2}\right) \hat{\mathbf{C}}\left(t, s_{1}\right)\right] d s_{1} d s_{2}$

$+\int_{0}^{t} L_{3}(t-s)[\hat{\mathbf{C}}(t, s) \mathbf{E} \otimes \mathbf{E}+\mathbf{E} \otimes \hat{\mathbf{C}}(t, s) \mathbf{E}] d s$

$+\int_{0}^{t} \int_{0}^{t} L_{4}\left(t-s_{1}, t-s_{2}\right)\left[\hat{\mathbf{C}}\left(t, s_{1}\right) \hat{\mathbf{C}}\left(t, s_{2}\right) \mathbf{E} \otimes \mathbf{E}+\mathbf{E} \otimes \hat{\mathbf{C}}\left(t, s_{1}\right) \hat{\mathbf{C}}\left(t, s_{2}\right) \mathbf{E}\right] d s_{1} d s_{2}$

$+\int_{0}^{t} L_{5}(t-s)[\hat{\mathbf{B}} \hat{\mathbf{C}}(t, s)+\hat{\mathbf{C}}(t, s) \hat{\mathbf{B}}] d s$

$+\int_{0}^{t} L_{6}(t-s)[\hat{\mathbf{B}} \hat{\mathbf{C}}(t, s) \mathbf{E} \otimes \mathbf{E}+\mathbf{E} \otimes \hat{\mathbf{B}} \hat{\mathbf{C}}(t, s) \mathbf{E}] d s$.

For times $t>t_{1}$, when the response is fluid-like, the response functional in (2.6) is assumed to have the form.

$$
\begin{aligned}
\Phi_{\text {fluid }}+p \mathbf{I} & =\hat{\boldsymbol{\Phi}}\left(t_{1}\right)+\hat{\beta}_{1} \mathbf{E} \otimes \mathbf{E}+\hat{\beta}_{2} \mathbf{D}+\int_{t_{1}}^{t} M_{1}(t-s) \hat{\mathbf{C}}(t, s) d s \\
& +\int_{t_{1}}^{t} \int_{t_{1}}^{t} M_{2}\left(t-s_{1}, t-s_{2}\right)\left[\hat{\mathbf{C}}\left(t . s_{1}\right) \hat{\mathbf{C}}\left(t, s_{2}\right)+\hat{\mathbf{C}}\left(t, s_{2}\right) \hat{\mathbf{C}}\left(t, s_{1}\right)\right] d s_{1} d s_{2} \\
& +\int_{t_{1}}^{t} M_{3}(t-s)[\hat{\mathbf{C}}(t, s) \mathbf{E} \otimes \mathbf{E}+\mathbf{E} \otimes \hat{\mathbf{C}}(t, s) \mathbf{E}] d s \\
& +\int_{t_{1}}^{t} \int_{t_{1}}^{t} M_{4}\left(t-s_{1}, t-s_{2}\right)\left[\hat{\mathbf{C}}\left(t, s_{1}\right) \hat{\mathbf{C}}\left(t, s_{2}\right) \mathbf{E} \otimes \mathbf{E}\right. \\
& \left.+\mathbf{E} \otimes \hat{\mathbf{C}}\left(t, s_{1}\right) \hat{\mathbf{C}}\left(t, s_{2}\right) \mathbf{E}\right] d s_{1} d s_{2} .
\end{aligned}
$$


In (3.1) and (3.2), $\mathbf{E}=\mathbf{E}(t), \hat{\mathbf{B}}=\mathbf{B}\left(t_{0}, t\right)-\mathbf{I}$ and $\hat{\mathbf{C}}(t, s)=\mathbf{C}(t, s)-\mathbf{I}$. The stretching tensor $\mathbf{D}$ arises when response functionals $\Lambda$ depend on $\hat{\mathbf{C}}(t, s)$ through the operation $\mathbf{D}=(\partial \hat{\mathbf{C}}(t, s) / \partial s)_{s=t}$. When the response functional in (3.1) contains the term in $\mathbf{D}$, the solid-like response has qualities of a Kelvin solid, e.g. no instantaneous elasticity. Scalars $\beta_{1}, \beta_{2}, \ldots, \beta_{7}, \hat{\beta}_{1}, \hat{\beta}_{2}$ are constants. Functions $L_{1}, L_{2}, \ldots, L_{6}, M_{1}, \ldots, M_{4}$ depend only on the arguments shown. It is further assumed that (a) these are continuous functions, (b) $\left|L_{i}\right|$ and $\left|M_{i}\right|$ monotonically decrease, (c) there is an $x_{0}>0$ such that if $x>x_{0}, x_{1}>x_{0}$, $x_{2}>x_{0}$, then $L_{i}(x)=0, M_{i}(x)=0, L_{i}\left(x_{1}, x_{2}\right)=0, M_{i}\left(x_{1}, x_{2}\right)=0$, for appropriate choices of the index $i$.

Each term in (3.1) has a specific purpose, each has the ability to capture a certain physical response observed in these materials. The terms $\hat{\mathbf{B}} \mathbf{E} \otimes \mathbf{E}$ and $\hat{\mathbf{B}}^{2} \mathbf{E} \otimes \mathbf{E}$ both reflect the contributions of the electric field to the shear stress, the former depending linearly on the shear strain and the latter nonlinearly. Thus, if experiments suggest a nonlinear dependence of the shear stress on the shear strain, then the term $\hat{\mathbf{B}} \mathbf{E} \otimes \mathbf{E}$ would not suffice. The term $\mathbf{D E} \otimes \mathbf{E}$ is included to model rate effects. Of course, if the rate effects are of higher order, we would need additional terms like $\mathbf{D}^{2} \mathbf{E} \otimes \mathbf{E}$. The term $\mathbf{E} \otimes \mathbf{E}$ represents a contribution to the stress purely due to the electric field. This models the normal stress induced due to the formation of particulate like chains in the direction of the electric field. The integral terms are selected to account for stress relaxation and transient response associated with start up flows and the frequency dependence of the response associated with steady state sinusoidal shearing. They also allow for the inclusion of the nonlinearities arising with variations in the electric field strength and the amplitude of the shear oscillations. The model can also incorporate transient response of the electrorheological materials when subjected to step changes in the electric field. The double integral terms allow for overtones in the stress response due to sinusoidal shear, which the single integral terms cannot. The integrands $\hat{\mathbf{C}}(t, s) \mathbf{E} \otimes \mathbf{E}$ and $\hat{\mathbf{C}}\left(t, s_{1}\right) \hat{\mathbf{C}}\left(t, s_{2}\right) \mathbf{E} \otimes \mathbf{E}$ serve the same purpose for the transient response as do $\hat{\mathbf{B}} \mathbf{E} \otimes \mathbf{E}$ and $\hat{\mathbf{B}}^{2} \mathbf{E} \otimes \mathbf{E}$ for the steady state response.

\section{Shear of ER materials}

Consider an ER material which is undergoing homogeneous simple shearing motion in the presence of an electric field. The motion will be described in a Cartesian coordinate system in which $X_{i}$ denote the coordinates of a particle in its initial state and $x_{i}(t)$ denote its coordinates at time $t$. The motion is described by the relations

$$
\begin{aligned}
& x_{1}(t)=X_{1}+K(t) X_{2}, \\
& x_{2}(t)=X_{2}, \quad x_{3}(t)=X_{3} .
\end{aligned}
$$


The variation of the shear $K(t)$ with time is arbitrary. The electric field vector is normal to the direction of particle motion and is given by

$E_{1}=E_{3}=0, \quad E_{2}(t)=E(t)$,

where $E(t)$ is assumed to be arbitrary.

The following descriptions of the shear motion are needed in order to calculate the matrix arguments in (3.1) and (3.2):

Particle position at time $s \leq t$ in terms of the particle position at time $t$

$x_{1}(s)=x_{1}(t)+[K(s)-K(t)] x_{2}(t)$,

$x_{2}(s)=x_{2}(t), \quad x_{3}(s)=x_{3}(t)$,

velocity field

$v_{1}(t)=\dot{K}(t) x_{2}(t), \quad v_{2}(t)=v_{3}(t)=0$,

where the superposed dot denotes differentiation with respect to time.

For notational convenience, let $\mathbf{F}=\mathbf{F}(0, t), \mathbf{B}=\mathbf{B}(0, t), K=K(t), E=E(t)$ and $K(t, s)=K(s)-K(t)$. Then, from (4.1)

$\mathbf{F}(0, t)=\mathbf{F}=\left[\begin{array}{lll}1 & K & 0 \\ 0 & 1 & 0 \\ 0 & 0 & 1\end{array}\right]$

The relative deformation gradient $\mathbf{F}(t, s)$ is obtained from (4.3),

$\mathbf{F}(t, s)=\left[\begin{array}{ccc}1 & K(t, s) & 0 \\ 0 & 1 & 0 \\ 0 & 0 & 1\end{array}\right]$

and from (4.4), the velocity gradient $\mathbf{L}$ is

$\mathbf{L}=\left[\begin{array}{ccc}0 & \dot{K} & 0 \\ 0 & 0 & 0 \\ 0 & 0 & 0\end{array}\right]$

Since $\hat{\mathbf{B}}=\mathbf{B}-\mathbf{I}=\mathbf{F F}^{T}-\mathbf{I}$,

$\hat{\mathbf{B}}=\left[\begin{array}{ccc}K^{2} & K & 0 \\ K & 0 & 0 \\ 0 & 0 & 0\end{array}\right], \quad \hat{\mathbf{B}}^{2}=\left[\begin{array}{ccc}K^{2}+K^{4} & K^{3} & 0 \\ K^{3} & K^{2} & 0 \\ 0 & 0 & 0\end{array}\right]$ 
By (4.7),

$\mathbf{D}=\frac{1}{2}\left(\mathbf{L}+\mathbf{L}^{T}\right)=\frac{1}{2}\left[\begin{array}{ccc}0 & \dot{K} & 0 \\ \dot{K} & 0 & 0 \\ 0 & 0 & 0\end{array}\right]$

By (4.6), $\hat{\mathbf{C}}(t, s)=\hat{\mathbf{C}}(t, s)-\mathbf{I}=\mathbf{F}(t, s)^{T} \mathbf{F}(t, s)-\mathbf{I}$ is given by

$$
\begin{aligned}
\hat{\mathbf{C}}(t, s) & =\left[\begin{array}{ccc}
0 & K(t, s) & 0 \\
K(t, s) & K^{2}(t, s) & 0 \\
0 & 0 & 0
\end{array}\right], \\
\hat{\mathbf{C}}\left(t, s_{1}\right) \hat{\mathbf{C}}\left(t, s_{2}\right) & =\left[\begin{array}{ccc}
K_{1} K_{2} & K_{1} K_{2}^{2} & 0 \\
K_{1}^{2} K_{2} & K_{1} K_{2}+K_{1}^{2} K_{2}^{2} & 0 \\
0 & 0 & 0
\end{array}\right],
\end{aligned}
$$

in which $K_{1}=K\left(t, s_{1}\right), K_{2}=K\left(t, s_{2}\right)$. The matrices which appear in (3.1) and (3.2) are given by (4.8) - (4.10) and the following:

By (4.2)

$\mathbf{E} \otimes \mathbf{E}=\left[\begin{array}{ccc}0 & 0 & 0 \\ 0 & E^{2} & 0 \\ 0 & 0 & 0\end{array}\right]$

$\hat{\mathbf{B}} \mathbf{E} \otimes \mathbf{E}=\left[\begin{array}{ccc}0 & K E^{2} & 0 \\ 0 & 0 & 0 \\ 0 & 0 & 0\end{array}\right], \quad \hat{\mathbf{B}}^{2} \mathbf{E} \otimes \mathbf{E}=\left[\begin{array}{ccc}0 & K^{3} E^{2} & 0 \\ 0 & K^{2} E^{2} & 0 \\ 0 & 0 & 0\end{array}\right]$,

$\mathbf{D E} \otimes \mathbf{E}=\left[\begin{array}{ccc}0 & \frac{1}{2} \dot{K} E^{2} & 0 \\ 0 & 0 & 0 \\ 0 & 0 & 0\end{array}\right]$

$\hat{\mathbf{C}}(t, s) \mathbf{E} \otimes \mathbf{E}==\left[\begin{array}{ccc}0 & K(t, s) E^{2} & 0 \\ 0 & K^{2}(t, s) E^{2} & 0 \\ 0 & 0 & 0\end{array}\right]$,

$\hat{\mathbf{C}}\left(t, s_{1}\right) \hat{\mathbf{C}}\left(t, s_{2}\right) \mathbf{E} \otimes \mathbf{E}=\left[\begin{array}{ccc}0 & K_{1} K_{2}^{2} E^{2} & 0 \\ 0 & \left(K_{1} K_{2}+K_{1}^{2} K_{2}^{2}\right) E^{2} & 0 \\ 0 & 0 & 0\end{array}\right]$, 
$\hat{\mathbf{B}} \hat{\mathbf{C}}(t, s)=\left[\begin{array}{ccc}K K(t, s) & K^{2} K(t, s)+K K^{2}(t, s) & 0 \\ 0 & K K(t, s) & 0 \\ 0 & 0 & 0\end{array}\right]$,

$\hat{\mathbf{B}} \hat{\mathbf{C}}(t, s) \mathbf{E} \otimes \mathbf{E}=\left[\begin{array}{ccc}0 & \left(K^{2} K(t, s)+K K^{2}(t, s)\right) E^{2} & 0 \\ 0 & K K(t, s) E^{2} & 0 \\ 0 & 0 & 0\end{array}\right]$

By (3.1) and (4.8)-(4.17), the stress components are, for $t \leq t_{1}$

$$
\begin{aligned}
\sigma_{11}+p= & \left(\beta_{2}+\beta_{3}\right) K^{2}+\beta_{3} K^{4}+2 \int_{0}^{t} \int_{0}^{t} L_{2}\left(t-s_{1}, t-s_{2}\right) K\left(t, s_{1}\right) K\left(t, s_{2}\right) d s_{1} d s_{2} \\
& +2 K \int_{0}^{t} L_{5}(t-s) K(t, s) d s, \\
\sigma_{22}+p= & \beta_{1} E^{2}+\left(\beta_{3}+2 \beta_{6} E^{2}\right) K^{2}+\int_{0}^{t}\left[L_{1}(t-s)+2 E^{2} L_{3}(t-s)\right] K^{2}(t, s) d s \\
& +2 \int_{0}^{t} \int_{0}^{t}\left[L_{2}\left(t-s_{1}, t-s_{2}\right)+E^{2} L_{4}\left(t-s_{1}, t-s_{2}\right)\right]\left[K\left(t, s_{1}\right) K\left(t, s_{2}\right)\right. \\
& \left.+K^{2}\left(t, s_{1}\right) K^{2}\left(t, s_{2}\right)\right] d s_{1} d s_{2} \\
& +2 K \int_{0}^{t}\left[L_{5}(t-s)+E^{2} L_{6}(t-s)\right] K(t, s) d s,
\end{aligned}
$$

$$
\begin{aligned}
\sigma_{12}= & \left(\beta_{2}+E^{2} \beta_{5}\right) K+\left(\beta_{3}+E^{2} \beta_{6}\right) K^{3}+\frac{1}{2}\left(\beta_{4}+E^{2} \beta_{7}\right) \dot{K} \\
& +\int_{0}^{t}\left[L_{1}(t-s)+E^{2} L_{3}(t-s)\right] K(t, s) d s \\
& +\int_{0}^{t}\left[L_{5}(t-s)+E^{2} L_{6}(t-s)\right]\left[K^{2} K(t, s)+K K^{2}(t, s)\right] d s \\
& +\int_{0}^{t} \int_{0}^{t}\left[L_{2}\left(t-s_{1}, t-s_{2}\right)+E^{2} L_{4}\left(t-s_{1}, t-s_{2}\right)\right]\left[K\left(t, s_{1}\right) K^{2}\left(t, s_{2}\right)\right. \\
& \left.+K^{2}\left(t, s_{1}\right) K\left(t, s_{2}\right)\right] d s_{1} d s_{2}
\end{aligned}
$$

By (3.2), (4.9) - (4.11) and (4.13) - (4.15), the stress components for $t>t_{1}$ are

$$
\begin{aligned}
\sigma_{11}+p= & \Phi_{11}\left(t_{1}\right)+2 \int_{t_{1}}^{t} \int_{t_{1}}^{t} M_{2}\left(t-s_{1}, t-s_{2}\right) K\left(t, s_{1}\right) K\left(t, s_{2}\right) d s_{1} d s_{2} \\
\sigma_{22}+p= & \hat{\Phi}_{22}\left(t_{1}\right)+\hat{\beta}_{1} E^{2}+\int_{t_{1}}^{t}\left[M_{1}(t-s)+2 E^{2} M_{3}(t-s)\right] K^{2}(t, s) d s \\
& +2 \int_{t_{1}}^{t} \int_{t_{1}}^{t}\left[M_{2}\left(t-s_{1}, t-s_{2}\right)+E^{2} M_{4}\left(t-s_{1}, t-s_{2}\right)\right]\left[K\left(t, s_{1}\right) K\left(t, s_{2}\right)\right. \\
& \left.+K^{2}\left(t, s_{1}\right) K^{2}\left(t, s_{2}\right)\right] d s_{1} d s_{2}
\end{aligned}
$$




$$
\begin{aligned}
\sigma_{12}= & \hat{\Phi}_{12}\left(t_{1}\right)+\frac{1}{2} \hat{\beta}_{2} \dot{K}+\int_{t_{1}}^{t}\left[M_{1}(t-s)+E^{2} M_{3}(t-s)\right] K(t, s) d s \\
+ & \int_{t_{1}}^{t} \int_{t_{1}}^{t}\left[M_{2}\left(t-s_{1}, t-s_{2}\right)+E^{2} M_{4}\left(t-s_{1}, t-s_{2}\right)\right]\left[K\left(t, s_{1}\right) K^{2}\left(t, s_{2}\right)\right. \\
& \left.+K^{2}\left(t, s_{1}\right) K\left(t, s_{2}\right)\right] d s_{1} d s_{2} .
\end{aligned}
$$

In addition, it is found that $\sigma_{33}+p=\sigma_{13}=\sigma_{23}=0$ for all times $t$.

\section{Constant shear rate start up motion}

Consider the motion in (4.1) in which the ER material starts from a state of rest at a constant shear rate,

$$
\begin{aligned}
K(t) & =0, \quad t<0, \\
& =\gamma_{0} t, \quad t \geq 0 .
\end{aligned}
$$

Then

$K(t, s)=\gamma_{0}(s-t), \quad \dot{K}(t)=\gamma_{0}$.

Initially when the material is solid-like, the shear stress is given by (4.20). Upon substituting (5.1) and (5.2) into (4.20), and an appropriate change in variables in the integrals, the expression for the shear stress becomes

$$
\sigma_{12}=\gamma_{0} \mu_{1}\left(E^{2}(t), t\right)+\gamma_{0}^{3} \mu_{2}\left(E^{2}(t), t\right),
$$

in which

$$
\begin{aligned}
\mu_{1}\left(E^{2}, t\right)= & \left(\beta_{2}+E^{2} \beta_{5}\right) t+\frac{1}{2}\left(\beta_{4}+E^{2} \beta_{7}\right)-\int_{0}^{t}\left[L_{1}(x)+E^{2} L_{3}(x)\right] x d x \\
\mu_{2}\left(E^{2}, t\right)= & \left(\beta_{3}+E^{2} \beta_{6}\right) t^{3}+\int_{0}^{t}\left[L_{5}(x)+E^{2} L_{6}(x)\right]\left(-t^{2} x+t x^{2}\right) d x \\
& -\int_{0}^{t} \int_{0}^{t}\left[L_{2}\left(x_{1}, x_{2}\right)+E^{2} L_{4}\left(x_{1}, x_{2}\right)\right] x_{1} x_{2}\left(x_{1}+x_{2}\right) d x_{1} d x_{2} .
\end{aligned}
$$

The normal stress differences are given by

$$
\sigma_{11}-\sigma_{33}=\gamma_{0}^{2} N_{11}(t)+\gamma_{0}^{4} N_{12}(t),
$$


where

$$
\begin{aligned}
N_{11}(t)= & \left(\beta_{2}+\beta_{3}\right) t^{2}-2 t \int_{0}^{t} L_{5}(x) x d x \\
& +2 \int_{0}^{t} \int_{0}^{t} L_{2}\left(x_{1}, x_{2}\right) x_{1} x_{2} d x_{1} d x_{2},
\end{aligned}
$$

$N_{12}(t)=\beta_{3} t^{4}$

and

$\sigma_{22}-\sigma_{33}=\beta_{1} E^{2}+\gamma_{0}^{2} N_{21}\left(E^{2}, t\right)+\gamma_{0}^{4} N_{22}\left(E^{2}, t\right)$

where

$$
\begin{aligned}
N_{21}\left(E^{2}, t\right)= & \left(\beta_{3}+2 E^{2} \beta_{6}\right) t^{2}+\int_{0}^{t}\left[L_{1}(x)+2 E^{2} L_{3}(x)\right] x^{2} d x \\
& -2 t \int_{0}^{t}\left[L_{5}(x)+E^{2} L_{6}(x)\right] x d x \\
& +2 \int_{0}^{t} \int_{0}^{t}\left[L_{2}\left(x_{1}, x_{2}\right)+E^{2} L_{4}\left(x_{1}, x_{2}\right)\right] x_{1} x_{2} d x_{1} d x_{2},
\end{aligned}
$$

$N_{22}\left(E^{2}, t\right)=2 \int_{0}^{t} \int_{0}^{t}\left[L_{2}\left(x_{1}, x_{2}\right)+E^{2} L_{4}\left(x_{1}, x_{2}\right)\right] x_{1}^{2} x_{2}^{2} d x_{1} d x_{2}$.

It is assumed that the stresses in the ER material grow with time and at some time $t_{1}$, the transition condition (2.2a) is satisfied. The stress components are then given by $(4.21)-(4.23)$. Let us consider the shear stress first. Upon substituting (5.2) into (4.23) and introducing a change of variables in the integrals, it is found that

$\sigma_{12}=\gamma_{0} \hat{\mu}_{1}\left(E^{2}(t), t, t_{1}\right)+\gamma_{0}^{3} \hat{\mu}_{2}\left(E^{2}(t), t, t_{1}\right)$

where

$$
\begin{gathered}
\hat{\mu}_{1}\left(E^{2}, t, t_{1}\right)=\mu_{1}\left(E^{2}, t_{1}\right)+\frac{1}{2} \hat{\beta}_{2}-\int_{0}^{t-t_{1}}\left[M_{1}(x)+E^{2} M_{3}(x)\right] x d x \\
\hat{\mu}_{2}\left(E^{2}, t, t_{1}\right)=\mu_{2}\left(E^{2}, t_{1}\right)-\int_{0}^{t-t_{1}} \int_{0}^{t-t_{1}}\left[M_{2}\left(x_{1}, x_{2}\right)+E^{2} M_{4}\left(x_{1}, x_{2}\right)\right] . \\
\cdot x_{1} x_{2}\left(x_{1}+x_{2}\right) d x_{1} d x_{2} .
\end{gathered}
$$

As time increases, the properties of $M_{i}, i=1,2,3,4$ are such that the integrals reach fixed values at $t=t_{1}+x_{0}$. The shear stress becomes independent of time 
$t$ and is given by

$$
\sigma_{12}=\mu\left(\gamma_{0}^{2}, E^{2}\right) \gamma_{0}
$$

where

$$
\begin{aligned}
\mu\left(\gamma_{0}^{2}, E^{2}\right)= & \mu_{1}\left(E^{2}, t_{1}\right)+\frac{1}{2} \hat{\beta}_{2}-\int_{0}^{x_{0}}\left[M_{1}(x)+E^{2} M_{3}(x)\right] x d x \\
& +\gamma_{0}^{2}\left[\mu_{2}\left(E^{2}, t_{1}\right)-\int_{0}^{x_{0}} \int_{0}^{x_{0}}\left[M_{2}\left(x_{1}, x_{2}\right)+E^{2} M_{4}\left(x_{1}, x_{2}\right)\right] .\right. \\
& \left.\cdot x_{1} x_{2}\left(x_{1}+x_{2}\right) d x_{1} d x_{2}\right] .
\end{aligned}
$$

$\mu\left(\gamma_{0}^{2}, E^{2}\right)$ represents a viscosity which depends on the shear rate $\gamma_{0}$ and electric field $E$.

Expressions for the normal stress differences can also be calculated. As with the shear stress, the normal stress differences become independent of time and are given as

$$
\begin{aligned}
& \sigma_{11}-\sigma_{33}= \gamma_{0}^{2}\left[N_{11}\left(E^{2}, t_{1}\right)+2 \int_{0}^{x_{0}} \int_{0}^{x_{0}} M_{2}\left(x_{1} x_{2}\right) x_{1} x_{2} d x_{1} d x_{2}\right]+\gamma_{0}^{4} N_{12}\left(E^{2}, t\right) \\
&= N_{1}\left(\gamma_{0}^{2}, E^{2}, t_{1}\right) \\
& \sigma_{22}-\sigma_{33}=\left(\beta_{1}+\hat{\beta}_{1}\right) E^{2}+\gamma_{0}^{2}\left[N_{21}\left(E^{2}(t), t\right)+\int_{0}^{x_{0}}\left[M_{1}(x)+2 E^{2} M_{3}(x)\right] x^{2} d x\right. \\
& \\
&\left.\quad+2 \int_{0}^{x_{0}} \int_{0}^{x_{0}}\left[M_{2}\left(x_{1}, x_{2}\right)+E^{2} M_{4}\left(x_{1}, x_{2}\right)\right] x_{1} x_{2} d x_{1} d x_{2}\right] \\
& \quad+\gamma_{0}^{4}\left[N_{22}\left(E^{2}(t), t_{1}\right)+2 \int_{0}^{x_{0}} \int_{0}^{x_{0}}\left[M_{2}\left(x_{1}, x_{2}\right)+E^{2} M_{4}\left(x_{1}, x_{2}\right)\right] .\right. \\
&\left.\quad \cdot x_{1}^{2} x_{2}^{2} d x_{1} d x_{2}\right] \\
&=N_{2}\left(\gamma_{0}^{2}, E^{2}, t_{1}\right) .
\end{aligned}
$$

Recall that the general constitutive equation given by (2.4) and (2.6) depends on the instantaneous value of the electric field. Thus the relations derived in this section are independent of time variation of the electric field. In order to study the influence of $E(t)$ in this model, note that the viscosity, by (5.4), (5.5) and (5.16) can be written in the form

$\mu\left(\gamma_{0}^{2}, E^{2}\right)=\mu_{10}+E^{2} \mu_{11}+\gamma_{0}^{2}\left(\mu_{20}+E^{2} \mu_{21}\right)$,

where

$\mu_{10}=\beta_{2} t_{1}+\frac{1}{2} \beta_{4}-\int_{0}^{t_{1}} L_{1}(x) x d x+\frac{1}{2} \hat{\beta}_{2}-\int_{0}^{x_{0}} M_{1}(x) x d x$, 
$\mu_{11}=\beta_{5} t_{1}+\frac{1}{2} \beta_{7}-\int_{0}^{t_{1}} L_{3}(x) x d x-\int_{0}^{x_{0}} M_{3}(x) x d x$

$$
\begin{aligned}
\mu_{20}= & \beta_{3} t_{1}^{3}+\int_{0}^{t_{1}} L_{5}(x)\left(-t_{1}^{2} x+t_{1} x^{2}\right) d x \\
& -\int_{0}^{t_{1}} \int_{0}^{t_{1}} L_{2}\left(x_{1}, x_{2}\right) x_{1} x_{2}\left(x_{1}+x_{2}\right) d x_{1} d x_{2} \\
& -\int_{0}^{x_{0}} \int_{0}^{x_{0}} M_{2}\left(x_{1}, x_{2}\right) x_{1} x_{2}\left(x_{1}+x_{2}\right) d x_{1} d x_{2},
\end{aligned}
$$

$$
\begin{aligned}
\mu_{21}= & \beta_{6} t_{1}^{3}+\int_{0}^{t_{1}} L_{6}(x)\left(-t_{1}^{2} x+t_{1} x^{2}\right) d x \\
& -\int_{0}^{t_{1}} \int_{0}^{t_{1}} L_{4}\left(x_{1}, x_{2}\right) x_{1} x_{2}\left(x_{1}+x_{2}\right) d x_{1} d x_{2} \\
& -\int_{0}^{x_{0}} \int_{0}^{x_{0}} M_{4}\left(x_{1}, x_{2}\right) x_{1} x_{2}\left(x_{1}+x_{2}\right) d x_{1} d x_{2} .
\end{aligned}
$$

When $E(t)=0$, the viscosity becomes $\mu_{10}+\gamma_{0}^{2} \mu_{20}$. Suppose that the material response is Newtonian. This is possible if $\beta_{2}=\beta_{3}=0, L_{1}(x)=M_{1}(x)=$ $L_{5}(x) \equiv 0, L_{2}\left(x_{1}, x_{2}\right)=M_{2}\left(x_{1}, x_{2}\right) \equiv 0$. Then $\mu\left(\gamma_{2}^{0}, 0\right)=\frac{1}{2}\left(\beta_{4}+\hat{\beta}_{2}\right)$.

Now let $E(t)=E_{0}$, a constant. Consider a sequence of shear flows at the same shear rate $\gamma_{0}$, but for increasing $E_{0}$. As $E_{0}$ increases, the terms in $\mu_{11}$ and $\mu_{21}$ begin to contribute to the viscosity. Referring to (3.1) and (3.2), as $E$ increases, the shear stress develops a dependence on strain tensor $\mathbf{B}$ through constant $\beta_{5}$ thereby introducing solid-like behavior. The stress also develops a dependence on the integrals, which introduce transient response. These terms could represent the growth of the shear stress to a constant value as shown by $\mathrm{Xu}$ and Liang [6]. Finally, the term involving $\mu_{21}$ increases, and the viscosity develops a dependence on shear rate. Analogous comments can be made about the normal stress differences. These will be omitted for the purpose of brevity.

Suppose steady-shear flow occurs in the presence of an oscillatory electric field, $E(t)=E_{0} \sin \omega t$. By (5.19)

$\mu\left(\gamma_{0}^{2}, E(t)^{2}\right)=\mu_{10}+\gamma_{0}^{2} \mu_{20}+E_{0}^{2} \frac{1-\cos 2 \omega t}{2}\left(\mu_{11}+\gamma_{0}^{2} \mu_{21}\right)$

The viscosity and hence the shear stress required to support the steady shear flow oscillate about a mean value with frequency $2 \omega$. As seen from (5.18), the normal stress differences also oscillate about a mean value with frequency $2 \omega$. This has implications for devices involving shear flow of ER materials. If the electric field vector is not constant, the fluid could exert time dependent normal stresses on the walls of the device, which could result in damage to the device and possibly undesirable acoustic effects. 


\section{Sinusoidally oscillating shear}

Consider the motion in (4.1) in which the ER material starts from a rest state and undergoes sinusoidally oscillating shear,

$$
\begin{array}{cc}
K(t)=0, & t<0, \\
K(t)=K_{0} \sin \omega t, & t \geq 0 .
\end{array}
$$

Let the shear amplitude be infinitesimal, $\left|K_{0}\right| \ll 1$, so that terms of order $K_{0}^{3}$ can be neglected in the expressions for $\sigma_{12}$. It will be assumed, for brevity of presentation, that the transition function satisfies (2.2b), e.g. the response of the ER material is in the solid-like regime.

On substitution of (6.1) into (4.20) neglecting terms of order $K_{0}^{3}$, and making the appropriate change of variable in the integrals, the shear stress $\sigma_{12}$ is found to be given by

$$
\sigma_{12}=K_{0}\left[\hat{G}_{1}\left(\omega, E^{2}, t\right) \sin \omega t+\hat{G}_{2}\left(\omega, E^{2}, t\right) \cos \omega t\right],
$$

where

$$
\begin{aligned}
\hat{G}_{1}\left(\omega, E^{2}, t\right)= & \beta_{2}+\int_{0}^{t} L_{1}(s)(\cos \omega s-1) d s \\
& +E^{2}\left[\beta_{5}+\int_{0}^{t} L_{3}(s)(\cos \omega s-1) d s\right],
\end{aligned}
$$

$$
\begin{aligned}
\hat{G}_{2}\left(\omega, E^{2}, t\right)= & \frac{1}{2} \beta_{4} \omega-\int_{0}^{t} L_{1}(s) \sin \omega s d s \\
& +E^{2}\left[\frac{1}{2} \beta_{7} \omega-\int_{0}^{t} L_{3}(s) \sin \omega s d s\right]
\end{aligned}
$$

Recalling the properties of $L_{1}(s), L_{3}(s)$, it is seen that the integrals in (6.3) and (6.4) become independent of time for $t>x_{0}$. The stress in (6.2) becomes

$\sigma_{12}=K_{0}\left[G_{1}\left(\omega, E^{2}\right) \sin \omega t+G_{2}\left(\omega, E^{2}\right) \cos \omega t\right)$,

where

$G_{\alpha}\left(\omega, E^{2}\right)=\hat{G}_{\alpha}\left(\omega, E^{2}, x_{0}\right), \quad \alpha=1,2$.

This result shows that the stress oscillates sinusoidally with frequency $\omega . G_{1}$ and $G_{2}$ correspond to the storage and loss moduli, respectively, of linear viscoelasticity, but depend on $E$. Note that

$\tan \delta\left(\omega, E^{2}\right)=\frac{G_{2}\left(\omega, E^{2}\right)}{G_{1}\left(\omega, E^{2}\right)}$, 
which shows that the phase angle also depends on frequency $\omega$ and electric field E.

As $K_{0}$ and/or $E$ increase, the terms of order $K_{0}^{3}$ in (4.20) become significant. It is straightforward, but tedious, to show that for $t>x_{0}$, the shear stress will consist of the terms in (6.5) plus an expression of the form

$K_{0}^{3}\left[\tilde{G}_{1}\left(\omega, E^{2}\right) \sin 3 \omega t+\tilde{G}_{2}\left(\omega, E^{2}\right) \cos 3 \omega t\right)$,

where $\tilde{G}_{1}$ and $\tilde{G}_{2}$ are of the form

$\tilde{G}_{\alpha}=\tilde{G}_{\alpha 0}(\omega)+E^{2} \tilde{G}_{\alpha 1}(\omega), \quad \alpha=1,2$.

Thus, higher harmonics of frequency $3 \omega$ appear in the shear stress response. According to (6.8), these can arise as $K_{0}$ increases. Note that if $\tilde{G}_{\alpha 0}=0$, $\alpha=1,2$, these terms are negligible if $E$ is small, but become significant as $E$ increases. This phenomenon has been shown in the experimental results of Gamota et al [11].

For each fixed electric field $E$, as $K_{0}$ increases, the stresses may increase so that the transition condition (2.2a) is met. It is then necessary to extend the discussion to account for (4.23). This adds to the complexity of the analysis, but the results will be analogous to that contained in (6.8). There will be higher harmonics in the shear stress, whose significance increases as $E$ increases.

\section{Concluding remarks}

Starting within the context of a very general frame work in which the stress in an electrorheological material exhibits solid-like and fluid-like behavior that depends on the history of the deformation gradient and the applied electric field, we have shown that complex non-linear effects are possible due to the interaction between the various kinematical tensors such as $\mathbf{B}, \mathbf{D}, \mathbf{C}(t, s)$ and $\mathbf{E}$. Using a more specific constitutive equation as an example, we are able to predict phenomena which correlate well with the observed response of electrorheological materials. Nonlinear phenomena like normal stress differences have been shown to be possible in the flow of electrorheological materials and the electrical field can affect the material properties in a non-linear fashion.

\section{Appendix}

The results of applying the representation method in [16] are presented first for $\Phi_{\text {fluid }}$ :

$\Phi_{\text {fluid }}=\alpha_{1} \mathbf{I}+\alpha_{2} \mathbf{E} \otimes \mathbf{E}+\sum_{m=1}^{N} \Lambda^{(m)}\left[\mathbf{J}^{(m)} ; I\right]$, 
where $\Lambda^{(m)}$ notes a functional which depends on the history of a symmetric matrix polynomial $\mathbf{J}^{(m)}$, and on a set of invariants $I . \alpha_{1}$ and $\alpha_{2}$ are scalar functionals which also depend on these invariants. The functional $\Lambda^{(m)}$ depends, as follows, on its matrix polynomial argument $\mathbf{J}^{(m)}$; if a component $J_{i j}^{(m)}$ can be written in the form, $J_{i j}^{(m)}=\varphi_{1} \hat{f}+\varphi_{2} \tilde{J}$, where $\varphi_{1}$ and $\varphi_{2}$ are constants and $\hat{J}, \tilde{J}$ depend on time, then

$\Lambda^{(m)}\left[\varphi_{1} \hat{J}+\varphi_{2} \tilde{J} ; I\right]=\varphi_{1} \Lambda^{(m)}[\hat{J} ; I]+\varphi_{2} \Lambda^{(m)}(\tilde{J} ; I)$.

The matrix polynominals are defined by

$\mathbf{J}^{(m)}=\frac{1}{2}\left[\mathbf{P}+\mathbf{P}^{T}\right]$

where the $\mathbf{P}$ fall into several groupings. The first depends on $\mathbf{C}(s, t)$, and its elements are

$\mathbf{Q}^{(m)}=\mathbf{C}\left(s_{1}, t\right) \mathbf{C}\left(s_{2}, t\right) \ldots \mathbf{C}\left(s_{m}, t\right), \quad m=1,2, \ldots, 5$

In the next group, each polynomial $\mathbf{P}$ depends on both $\mathbf{C}(s, t)$ and $\mathbf{E}(t)=\mathbf{E}$ through:

$\mathbf{Q}^{(q)} \mathbf{E} \otimes \mathbf{Q}^{(r)} \mathbf{E}, \quad q, r=0,1,2,3,4$,

$$
q+r \leq 4 \text {, }
$$

with $\mathbf{Q}^{(0)}=\mathbf{I}$.

The set of invariants $I$ consists of the trace of each polynomial $\mathbf{P}$ just defined. The polynomials $\mathbf{P}$ defined in (8.5) do not form the smallest possible set. Some may be expressible both in terms of others and some of the invariants of the set $I$. The polynomials are presented in this manner for convenience and brevity. A minimal set can be constructed using the method outlined in Spencer's article [16]. However, the purpose here is to present the general form of the response functional.

Each functional $\Lambda^{(m)}$ depends on all values of its matrix and invariant arguments as time variables $s_{i}$ vary in the interval $\left[t_{1}, t\right]$.

It is instructive to consider the following example which illustrates the operation defined in (8.2), and also emphasizes both the dependence on the history of $\mathbf{C}(s, t)$ and the dependence on the current value of $\mathbf{E}(t)$. For some functional $\Lambda^{(P)}$,

$$
\begin{aligned}
& \Lambda^{(P)}\left[\frac{1}{2}(\mathbf{C}(s, t) \mathbf{E}(t) \otimes \mathbf{E}(t)+\mathbf{E}(t) \otimes \mathbf{C}(s, t) \mathbf{E}(t)) ; I\right] \\
& =\frac{1}{2} \Lambda^{(P)}[\mathbf{C}(s, t) ; I] \mathbf{E}(t) \otimes \mathbf{E}(t)+\frac{1}{2} \mathbf{E}(t) \otimes \Lambda^{(P)}[\mathbf{C}(s, t) ; I] \mathbf{E} .
\end{aligned}
$$


In component form

$$
\begin{aligned}
& \Lambda^{(P)}\left[\frac{1}{2}\left(C_{i p}(s, t) E_{p}(t) E_{j}(t)+E_{i}(t) C_{j p}(s, t) E_{p}(t)\right) ; I\right] \\
& =\frac{1}{2}\left\{\Lambda^{(P)}\left[C_{i p}(s, t) ; I\right] E_{p}(t) E_{j}(t)+E_{i}(t) \Lambda^{(P)}\left[C_{j p}(s, t) ; I\right] E_{p}(t)\right\} .
\end{aligned}
$$

The response functional $\Phi_{\text {solid }}$ in (2.4) has the form

$$
\begin{aligned}
\Phi_{\text {solid }}= & \hat{\alpha}_{1} \mathbf{I}+\hat{\alpha}_{2} \mathbf{E} \otimes \mathbf{E}+\hat{\alpha}_{3} \mathbf{B}+\hat{\alpha}_{4} \mathbf{B}^{2} \\
& +\hat{\alpha}_{5}(\mathbf{B E} \otimes \mathbf{E}+\mathbf{E} \otimes \mathbf{B E})+\hat{\alpha}_{6}\left(\mathbf{B}^{2} \mathbf{E} \otimes \mathbf{E}+\mathbf{E} \otimes \mathbf{B}^{2} \mathbf{E}\right) \\
& +\sum_{m=1}^{\hat{N}} \hat{\Lambda}^{(m)}\left[\mathbf{J}^{(m)} ; \hat{I}\right],
\end{aligned}
$$

where $\hat{\Lambda}^{(m)}$ notes a functional which depends linearly on its matrix polynomial argument $\mathbf{J}^{(m)}$ and on a new set of invariants $\hat{I}$. $\hat{\alpha}_{1}, \hat{\alpha}_{2}, \ldots, \hat{\alpha}_{6}$ are scalar functionals of the invariants $\hat{I}$.

The matrix polynomials have the form (8.3), and can be organized into several groups. The first group depends only on $\mathbf{C}(s, t)$ and consists of those given as (8.4). Each polynomial in the second group depends on $\mathbf{C}(s, t)$ and $\mathbf{E}(t)$ and has the form in (8.5). The polynomials in the third group dependent on $\mathbf{C}(s, t)$ and $\mathbf{B}$ and have the form

$$
\begin{gathered}
\mathbf{P}^{(m)} \mathbf{B} \mathbf{P}^{(n)}, \quad \\
m, n=0,1,2,3,4 \\
m+n \leq 4,
\end{gathered}
$$

or

$$
\begin{gathered}
\mathbf{P}^{(m)} \mathbf{B}^{2} \mathbf{P}^{(n)}, \quad \\
m, n=0,1,2,3 \\
m+n \leq 3,
\end{gathered}
$$

or

$\mathbf{B}^{2} \mathbf{C}\left(s_{1}, t\right) \mathbf{B C}\left(s_{2}, t\right)$,

$\mathbf{C}\left(s_{1}, t\right) \mathbf{B}^{2} \mathbf{C}\left(s_{2}, t\right) \mathbf{B}$.

The last group depends on $\mathbf{C}(s, t), \mathbf{B}$ and $\mathbf{E}(t)$ and has the form

$$
\mathbf{P}^{(A)} \mathbf{E} \otimes \mathbf{P}^{(B)} \mathbf{E}
$$

where $\mathbf{P}^{(A)}, \mathbf{P}^{(B)}$ depend on $\mathbf{C}(s, t)$ and $\mathbf{B}$. The total degree of $\mathbf{P}^{(A)}$ and $\mathbf{P}^{(B)}$ must 
be less than or equal to 4 . Examples of such a matrix polynominal are $\mathbf{B C}\left(s_{1}, t\right) \mathbf{C}\left(s_{2}, t\right) \mathbf{C}\left(s_{3}, t\right) \mathbf{E} \otimes \mathbf{E}$,

$\mathbf{B C}\left(s_{1}, t\right) \mathbf{C}\left(s_{2}, t\right) \mathbf{E} \otimes \mathbf{C}\left(s_{3}, t\right) \mathbf{E}$.
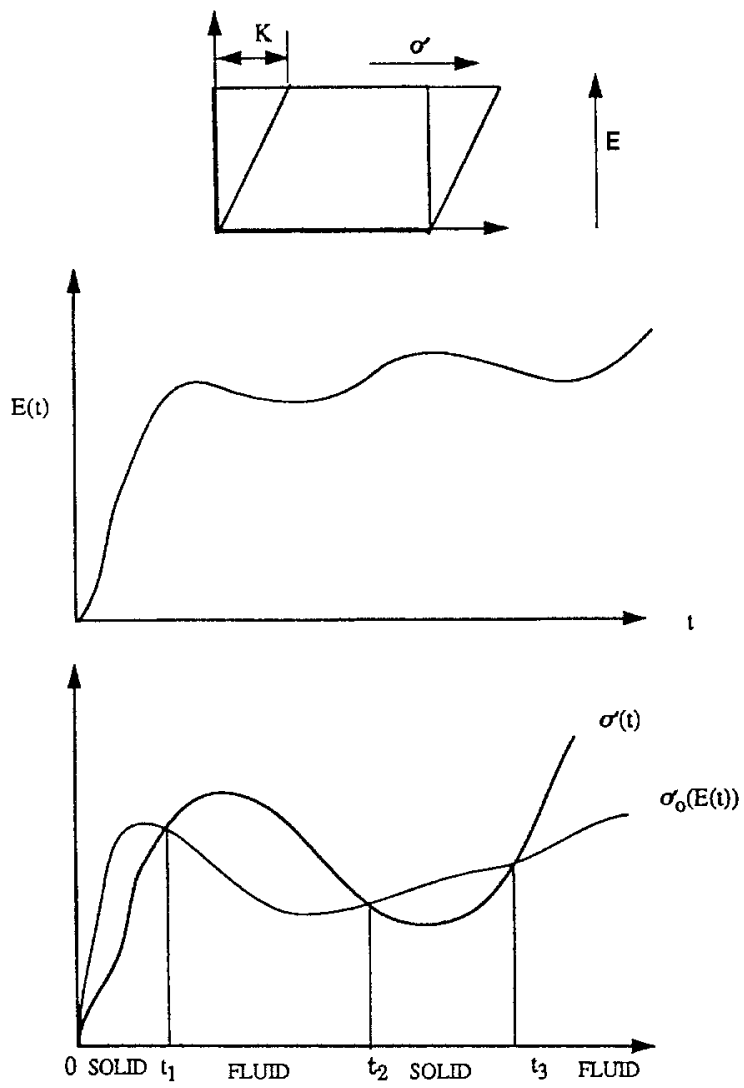

Fig. 1. Determination of time intervals for solid-like and fluid-like response for an electric field history $E(t)$, shear stress history $\sigma(t)$, and transition shear stress history $\sigma_{o}(t)$

\section{References}

1. Atkin, R. J.; Shi, X.; Bullough W. A.: "Solutions of the Constitutive Equations for the Flow of an Electrorheological Fluid in Radial Configurations", J. Rheology, 35 (1991) $1441-1461$

2. Rajagopal, K. R.; Wineman, A. S.: "Flow of Electro-Rheological Materials", Acta Mechanica, 91, (1992) 57-75

3. Brooks, B.; Goodwin, J.; Hjelm, C.; Marshall, L.; Zukoski, C.: "Viscoelastic Studies on an Electro-Rheological Fluid", Colloids Surf. 18 (1986) 293-312 
4. Vinogradov, G. V.; Shulman, Z. P.; Yanovskii, Y. G.; Barancheeva, V. V.; Korobko, E. V; Bukovich,I. V.: "Viscoelastic Behavior of Electro-Rheological Suspensions", Inzh.-Fiz, Zh. 50 ((1986)) 605-609

5. Thurston, G. B.; Gaertner, E. B.: "Viscoelasticity of Electrorheological Fluids During Oscillatory Flow in a Rectangular Channel", J. Rheol. 35 (1991) 1327-1343

6. Xu, Y.Z.; Liang, R. F.: "Electrorheological Properties of Semiconducting PolymerBased Suspensions", J. Rheol. 35 (1991) 1355-1373

7. Yen, W. S.; Achorn, P. J: "A Study of the Dynamic Behavior of an Electrorheological Fluid", J. Rheol., 35 (1991) 1375-1384

8. Gamota, D.R.; Filisko, F.E.: "Dynamic Mechanical Studies of Electrorheological Materials: Moderate Frequencies", J. Rheol. 35 (1991) 399-425

9. Gamota, D. R.; Filisko, F.E.: "High Frequency Dynamic Mechanical Study of an Aluminosilicate Electrorheological Material", J. Rheol. 35 (1991) 1411 - 1425

10. Jordan, T. C.; Shaw, M. T.; McLeish, T. C. B.: "Viscoelastic Response of Electrorheological Fluids. II: Field Strength and Strain Dependence", J. Rheol., 36, (1992) $441-463$

11. Gamota, D. R.; Wineman, A. S.; Filisko, F. E.: "Fourier Transform Analysis: Fundamental Nonlinear Dynamic Response of an Electrorheological Material", J. Rheol. 37, (1993), $919-934$

12. Conrad, H.; Sprecher, A.F. Choi, Y.; Chen, Y.; "The Temperature Dependence of the Electrical Properties and Strength of Electrorheological Fluids", J. Rheol., 35 (1991) $1393-1410$

13. Rajagopal, K.R.; Wineman, A.S.: "On Constitutive Equations for Branching of Response with Selectivity", Int. J. Non-Linear Mechanics, 15 (1980) 83-91

14. Wineman, A.S.; Rajagopal, K. R.: "On a Constitutive Theory for Materials Undergoing Microstructural Changes", Archives of Mechanics, 42 (1990) 53-74

15. Rajagopal, K. R.; Wineman A.S.: "A Constitutive Equation for Non-Linear Solids Which Undergo Deformation Induced Microstructural Changes", Int. J. Plasticity, 8 (1992) $385-395$

16. Spencer, A. J. M.: in Continuum Physics, ed. A. C. Eringen, vol. 3, Academic Press, New York (1975)

Alan S. Wineman

Department of Mechanical Engineering and Applied Mechanics

The University of Michigan

Ann Arbor, Michigan 48104

USA

K. Rajagopal

Department of Mechanical Engineering

University of Pittsburgh

Pittsburgh, PA 15261

USA

Received January 14, 1993 